\title{
Síndrome de Burnout em equipe de enfermagem que atua na urgência e emergência.
}

\section{Burnout syndrome in the nursing staff working in the urgency and emergency.}

\section{Síndrome de burnout en equipo de enfermería que actua en urgencia y emergencia.}

Hellen Raquel Oliveira de Sousa

RESUMO: O presente trabalho teve por objetivo Investigar a presença de sinais e sintomas de exaustão física e psicológica que possam indicar a ocorrência de síndrome de Burnout em profissionais da equipe de enfermagem da unidade de pronto socorro do Hospital das Clinicas da Universidade Federal de Goiás (HC/UFG). Métodos: Trata-se de um estudo exploratório com abordagem predominantemente qualitativa, no qual, os dados foram coletados através de entrevista semi-estruturada com a equipe de enfermagem. Resultados: $O$ estudo evidenciou que os sinais e sintomas mais freqüentes que a equipe de enfermagem apresentou foram as manifestações psicológicas, seguidas de outras manifestações, tais como sentimentos negativos e positivos. Conclusão: Outros aspectos relevantes foram encontrados como a necessidade de oficinas, atividades laborais e interações com o grupo gerando coleguismo e melhor interação da equipe, o gestor da unidade deve programar ações que gerem condições para que os sujeitos consigam exercer suas funções minimizando os fatores que levam ao esgotamento profissional e à síndrome. Descritores: Síndrome de Burnout, Enfermagem, sinais e sintomas, qualidade na assistência.

\begin{abstract}
Objective. The objective of this study was to investigate the presence of signs and symptoms of physical and psychological exhaustion that could indicate the occurrence of burnout syndrome in professionals of the nursing team of the emergency room of the Hospital das Clínicas of the Federal University of Goiás (HC / UFG) Methods. This is an exploratory study with a predominantly qualitative approach, in which data were collected through a semi-structured interview with the nursing team. Results. The study evidenced that the most frequent signs and symptoms that the nursing team presented were the psychological manifestations, followed by other manifestations, such as negative and positive feelings. Conclusion. Other relevant aspects were found such as the need for workshops, work activities and interactions with the group
\end{abstract}

$1^{1}$ Universidade Federal de Goiás 
generating teamwork and better team interaction, the unit manager should program actions that generate conditions so that the subjects can exercise their functions minimizing the factors that lead to the Exhaustion and the syndrome.

Descriptors: Burnout syndrome, Nursing, signs and symptoms, quality of care.

RESUMEN: Objetivo. Este estudio tuvo como objetivo investigar la presencia de signos y síntomas de agotamiento físico y psicológico que podría indicar la presencia de burnout en unidad profesional del personal de enfermería del síndrome de la sala de urgencias del Hospital de Clínicas de la Universidad Federal de Goiás (HC / UFG) métodos. Se trata de un estudio exploratorio con enfoque cualitativo, en el que se recogieron los datos a través de semiestructurada con la entrevista personal de enfermería. Resultados. El estudio mostró que los signos más frecuentes y síntomas que el personal de enfermería había sido las manifestaciones psicológicas, seguidos de otros eventos, como los sentimientos negativos y positivos.Conclusão. Otro aspecto relevante encontró la necesidad de talleres, actividades de trabajo y las interacciones con la colegialidad de generación de grupo y una mejor interacción del equipo, el gerente de la unidad debe programar acciones que crean las condiciones para que los sujetos son capaces de ejercer sus funciones, minimizando los factores que conducen a el agotamiento y el síndrome.

Palabras clave: Síndrome de Burnout, enfermería, signos y síntomas, calidad de la atención.

\section{INTRODUÇÃO}

As transformações políticas, sociais, econômicos, organizacionais e culturais carregam consigo novas exigências e modelos de trabalho gerando situações que influenciam diretamente no processo de saúde e doença da sociedade ${ }^{1}$.

A equipe de enfermagem, inserida em uma sociedade capitalista onde cada dia surgem novos ideais, preocupa-se em cumprir suas tarefas e exigências. Para isto, muitas vezes, vincula-se a duas ou mais instituições para desenvolver suas atividades laborais, o que se somando ao perfil do serviço de saúde gera sobrecarga de trabalho e de estresse afetando diretamente o comportamento físico bio-psico-social do profissional, a qualidade de vida e da assistência de enfermagem ${ }^{1}$.

Considerando o ser humano como sujeito autônomo em uma unidade de corpo e mente que reage de acordo com suas vivências, preocupa o fato de que dentro da própria unidade de saúde profissionais têm apresentado surtos de estresse, alterações no comportamento, sinais de exaustão e muitos atestados médico ${ }^{2}$.

A presença da síndrome no mundo esta cada dia mais evidente, em Portugal a cada quatro enfermeiros um apresentava Burnout ${ }^{3}$. No Brasil, alguns estudos mostram um perfil de risco constituído por mulheres e jovens que possuem uma profissão estressante, associado à carga horária e condições de trabalho e dificuldade de conciliar trabalho e família ${ }^{4}$. Pesquisa realizada 
por Jodas e Haddad ${ }^{5}$ no Pronto Socorro do Hospital Universitário Regional do Norte do Paraná (HURNP) investigou sinais e sintomas de Burnout em trabalhadores de enfermagem com fatores preditores. O estudo evidenciou que $8,2 \%$ dos profissionais estavam em estado de Burnout, ou seja, apresentavam os sinais e sintomas da doença e 54,1\% apresentavam predisposição para desenvolver esse problema. Como se percebe por meio dos estudos realizados a predisposição para o desenvolvimento da Síndrome de Burnout acomete grande parte dos trabalhadores da equipe de saúde, o que pode ser atribuído à alta carga de estresse a qual os mesmos estão expostos em decorrência do tipo de suas atividades profissionais. Na área de urgência e emergência, as situações de estresses podem ocorrer ainda com mais freqüência uma vez que, as prestações de serviços em caráter imediato e mediato os colocam na obrigação de tomarem decisões, muitas vezes, sem tempo suficiente para pensar sobre as mesmas.

De acordo com estudos realizados na área de urgência e emergência e por meio de observações quanto ao aparecimento de queixas de funcionários sobre cansaço físico e dificuldades para controlar as emoções, este assunto passou a fazer parte de um amplo campo de interesse uma vez que, sintomas como os apresentados pelas equipes de saúde, poderiam interferir na assistência de enfermagem prestada aos clientes. Segundo a literatura e pesquisas recentes, tais queixas apresentadas podem ser reflexos de sinais e sintomas de exaustão física e psicológica. Isto nos leva a questionar se estes sinais e sintomas poderiam estar relacionadas com a síndrome de Burnout. Como estes poderiam interferir no desempenho das atividades de enfermagem dos profissionais? E, quais as situações poderiam interferir na ocorrência dos mesmos?

Os resultados deste trabalho podem proporcionar benefícios que abrangem desde o paciente, a comunidade, a equipe de enfermagem a academia, até a instituição de saúde, uma vez que, a definição de um diagnóstico relacionado à saúde dos profissionais deste setor da instituição fornecerá informações aos gestores que subsidiará a elaboração de plano de cuidado à saúde dos mesmos mantendo assim a qualidade do serviço prestado à comunidade. Além disso, o conhecimento produzido ainda poderá ser utilizado na formação acadêmica dos futuros profissionais de enfermagem.

Assim o presente trabalho teve por objetivo investigar a presença de sinais e sintomas de exaustão física e psicológica que possam indicar a ocorrência de síndrome de Burnout em profissionais da equipe de enfermagem da unidade de pronto socorro do Hospital das Clinicas da Universidade Federal de Goiás (HC/UFG).

\section{MÉTODO}

Trata-se de um estudo descritivo com abordagem qualitativa. O estudo descritivo é definido como um levantamento sistemático dos problemas, características ou fenômenos observados da população estudada ${ }^{6} \mathrm{O}$ estudo foi realizado na Unidade de Pronto Atendimento do Hospital das Clinicas HC/ UFG que é composta pela Seção de Enfermagem em Acolhimento, Setor de Urgência

ISSN 1982-8829 Tempus, actas de saúde colet, Brasília, 11(4), 185-196, dez, 2017. Epub Ago/2018 
e Emergência Adulto e Setor de Enfermagem Urgência e Emergência Pediátrico (SERUPE). Atendendo aos critérios de inclusão e exclusão, este estudo obteve 31 participantes, sendo nove enfermeiros e 22 técnicos de enfermagem. Foram excluídos 38 profissionais, 12 que estavam de férias, sete de licença médica, dois se negaram a participar do estudo e 17 por desencontros em função de readaptações na escala e faltas. A coleta de dados foi realizada por meio de entrevista gravada e escrita, sendo esta ultima utilizada quando o participante do estudo não se sentia à vontade para responder por meio de gravação. Assim 13 participantes responderam à pesquisa com o auxílio de um gravador, sendo as informações posteriormente, transcritas pela pesquisadora e validadas pelos participantes, e os outros 18 participantes, à medida que respondiam às perguntas, as respostas eram registradas manualmente pela pesquisadora.

Para direcionar a coleta de dados foi utilizado um instrumento, elaborado pela pesquisadora, com perguntas abertas que buscou identificar os sinais e sintomas de exaustão física e psicológica apresentados pelos profissionais em estudo A elaboração deste instrumento de coleta de dados teve como orientador o questionário proposto por Maslach e Jackson ${ }^{7}$ - Maslach Burnout Inventory (MBI) que contem questões cujas respostas buscaram responder aos objetivos propostos.

\section{RESULTADO E DISCUSSÃO}

Os dados sócioprofissiográficos mostraram que a maior parte dos participantes deste estudo constitui-se de categoria profissional de técnico de enfermagem (71\%) em detrimento do percentual de enfermeiros (29\%). No que se refere ao sexo observa-se a predominância de mulheres $(90,3 \%)$. Outra característica dos participantes deste estudo se refere à faixa etária a maior parte dos profissionais se encontrava entre 41 e 50 anos de idade (32,2\%), seguido pela faixa de 51 a 60 anos com percentuais de 29,1\%. A pesquisa evidenciou que 80,6 \% dos profissionais têm mais de 10 anos de profissão e $77,4 \%$ trabalham a mais de 10 anos na unidade de pronto socorro. Outra realidade encontrada foi que $51,6 \%$ dos participantes possuíam dois vínculos empregatícios.

Os sinais e sintomas mais freqüentes que a equipe de enfermagem apresentou foram manifestações psicológicas, seguidas de outras manifestações, tais como sentimentos negativos e positivos como cansaço excessivo e esperança.

\section{Manifestações físicas}

As manifestações de exaustão físicas mais significativas apresentadas pela equipe de enfermagem foram dores musculares, no pescoço e coluna, cefaléias e enxaquecas que obtiveram percentual $46,5 \%$ seguido de transtornos cardiovasculares e fadiga constante que representaram cada um 20,9\%. As manifestações físicas referentes ao distúrbio do sono apresentaram menor percentual, ou seja, 11,7\%. Estas manifestações são evidenciadas nos depoimentos que se seguem:

“Tenho dores, dormência na perna quando me esforço (...)" (TE14) 
"Quando me esforço sinto dores e dormência na perna e glúteo (...)" (TE15)

"Sou hipertensa e sinto dores na coluna" (TE6)

"Tenho enxaqueca, dores musculares e na coluna” (TE17)

As dores musculares, no pescoço e coluna, cefaléias e enxaquecas são sinais e sintomas presentes em outros tipos de doenças ou podem aparecer isoladamente, por isso muitos entrevistados além de relatarem o aparecimento de doenças também queixam de dores, o que pode justificar o maior percentual encontrado neste estudo.

A equipe de enfermagem está composta por um grupo significativo de profissionais que não se encontram na faixa etária de atividade laboral e por outro grupo que já está margeando a mesma. Além disso, observa-se ainda que grande parte da equipe já esta na profissão há mais de dez anos. Estas evidências deixam claro que a população de servidores esta envelhecendo e correlacionado a isto as doenças, sinais e sintomas típicos da idade avançada como: dores musculares, transtornos cardiovasculares, diminuição da acuidade visual e auditiva entre outros ${ }^{8}$. Assim sendo, estes dados são preocupantes, pois além das dificuldades com o perfil da profissão e da atividade exercida, ainda sofrem com os sintomas característicos do processo de envelhecimento prejudicando diretamente a qualidade das atividades prestadas pela equipe, e sobrecarregando outros funcionários mais jovens. O depoimento abaixo evidencia a fragilidade e dificuldade que estes sinais trazem:

“Tenho apenas 30\% da audição, faço leitura labial (...)” (TE10)

“Quando a equipe é formada por (...) (pessoas com restrições) aí sobra pra mim porque este não pode punsionar, aquele não fica na reanimação (...) aí eu canso mais (...)” (TE1)

O estudo demonstrou que $71,1 \%$ dos profissionais entrevistados possuem algum distúrbio de saúde ou aparecimento de doenças. Quanto ao uso de algum tipo de medicação, 51,6\% relataram usar alguma droga, dentre elas, anti-hipertensivo, hipnóticos, analgésicos, antidepressivos entre outros.

As falas abaixo são exemplos dessas referências.

“(...) quando fiquei hipertensa foi um período de muito estresse, tenho lesões no braço por esforço repetitivo, o médico do trabalho já falou (...) hoje estou readaptada" (TE6)

“Tenho dormências nos pés e dores no calcanhar e planta do pé, perdi parte da audição devido os choros e gritos das crianças, o trabalho me causa insônia (...)" (TE20)

O número de profissionais que relataram aparecimento de algum tipo de doença foi maior do que os relatos de uso de remédios. Isso pode se justificar uma vez que nem todos que apresentam doenças fizeram referência ao uso de medicações, sendo que quando mencionaram 
uso de medicamentos, esses eram usados para tratar de sintomas isolados ou das próprias doenças relatadas.

\section{Manifestações comportamentais}

Entende-se por manifestações comportamentais os sintomas que representam conduta do profissional no ambiente de trabalho ${ }^{9}$.

Dentre os entrevistados $74,2 \%$ apresentaram algum sintoma que indicasse alteração comportamental sendo os mais comuns o aumento dos conflitos com colegas, irritabilidade, incapacidade de relaxar, cumprimento irregular da carga horária e longas pausas para descanso cujos percentuais foram, respectivamente $32,2 \% ; 16,2 \% ; 16,2 \% ; 6,4 \%$ e $3,2 \%$. Os que não apresentaram queixas nesta categoria foram $25,8 \%$ dos participantes.

Estas manifestações são apresentadas nos depoimentos abaixo:

\footnotetext{
"Tenho me estressado com algumas pessoas depende da escala." (TE11).

"uma das sugestões seria os intervalos, agente trabalha doze horas e não tem horário de almoço, nunca consegui entender isso, agente almoça correndo e tem que voltar, precisamos no mínimo uma hora de almoço, depois agente vem para o repouso e não quer mais sair, mas aí tem que voltar e terminar o plantão.” (TE9).
}

Apesar do perfil do serviço exigir atividades em equipe esta categoria de sinais e sintomas demonstrou que o aumento de conflitos com colegas de equipe e irritabilidade trazem transtornos que podem gerar dificuldade na execução das tarefas diárias. Firme ${ }^{9}$ justifica afirmando que este comportamento é reflexo da exaustão emocional e despersonalização pelo esgotamento afetivo e estabelecimento de relações interpessoais de forma fria o que caracteriza a insensibilidade emocional.

\section{Manifestações psicológicas}

Manifestações psicológicas podem ser entendidas como um conjunto de sinais e sintomas que se relacionam com fenômenos mentais e emocionais ${ }^{9}$.

Os com maior evidência no estudo foram: falta de atenção, desânimo e diminuição da concentração com percentual de $10,9 \%$ seguidos por sentimento de impotência $(9,7 \%)$; frustração $(9,1 \%)$; desconfiança e baixa auto-estima $(9,1 \%)$; solidão, sentimento de alienação e depressão $(7,4 \%)$.

Uma vez que o perfil do serviço exige que o trabalho seja desempenhado em equipe, o sentimento de isolamento, solidão e impotência, apesar de não representar o maior percentual dos 
relatos, deve ser analisado por oferecer risco para o aparecimento de transtornos mentais graves e potencializar a execução do trabalho de forma individualizada causando, aos trabalhadores, sofrimento de várias ordens.

Estudo realizado sobre os fatores sociais associados a transtornos mentais com situações de risco na atenção primária de saúde, ressaltam que

Para o sujeito acometido, a qualidade de vida fica limitada, o que pode produzir isolamento e exclusão social. Para manter o tratamento desses sujeitos, são necessárias ações intersetoriais e com pluralidade de profissionais da saúde. No Brasil, o Ministério da Saúde estima que 5 milhões de pessoas necessitem de cuidados contínuos em saúde mental, em função de acometimentos como: psicoses, transtornos de humor graves, neuroses graves e retardo mental grave. A importância desse cuidado é ratificada pela Agência Nacional de Saúde (ANS), órgão regulador em Saúde Suplementar no Brasil. A ANS não inclui (...) somente as psicoses funcionais, como: esquizofrenias, transtornos esquizotípicos, delirantes e transtornos de humor graves ${ }^{10}$.

Os depoimentos a seguir demonstram estes sintomas psicológicos:

"Em todos os momentos me sinto sozinha." (TE7)

"Dependendo da equipe eu trabalho sozinha" (TE1)

"Acho que, igual hoje muito cheio, muito puxado, sem leito pra ninguém, os pacientes ficam me pressionando, aí eu fico exausta emocionalmente, sem saber o que fazer (...) acho que poderia fazer mais se tivesse uma estrutura melhor." (E1)

\section{Manifestações defensivas}

Manifestações defensivas podem ser entendidas como sinais e sintomas que representam o afastamento psicológico do profissional de sua clientela e relações sociais ${ }^{11 .}$

Dentre as manifestações defensivas relatadas pela equipe de enfermagem destacou-se a perda de interesse pelo trabalho e culpa do mal estar no serviço que alcançaram percentuais de 33,8\%, seguido do sentimento de perda do interesse pelo lazer $(27,9)$. Para ilustrar esses achados, têm-se os depoimentos abaixo:

\footnotetext{
“Ás vezes, eu sinto desanimada, cansada, pensando em mudar de área” (E1)

“Minha vida social é um desastre.” (E1)

“(...) tenho pouco lazer, mas não tenho vontade (...).”(TE3)

“(...) meus problemas de saúde com certeza estão relacionados com o trabalho (...)” (TE6)
} 
“(...) os meus problemas de saúde tem relação como meu serviço completamente (...)” Eu não estou suportando vir para cá, quando eu penso que tenho que vir, da vontade de morrer, não quero vir, não quero falar com ninguém quero fazer o que tenho que fazer e ponto final, só isso (...). Ontem quando eu pensei em vir para cá, eu comecei a chorar, chorei até, em saber que tinha que vir, não quero vir. Eu não sei o que está acontecendo comigo, eu sinto uma completa indisposição, falta de alegria, desânimo, só de pensar nos colega aí que não quero vir mesmo, (...), não só pela equipe é tudo o contexto todo, os pacientes a rotina essa atividade repetitiva (...).”

O afastamento dos profissionais de sua clientela e das relações sociais impede que o profissional haja como orienta a Política Nacional de Humanização ${ }^{12}$ que através dos princípios e diretrizes, define que o acolhimento acontece durante a análise dos processos de trabalho com o objetivo de construir relações de confiança e compromisso, além do vínculo entre a equipe/serviço, trabalho/ equipe e usuário através de sua rede socioafetiva. Portanto, além das relações com a equipe a clientela também deve fazer parte desta rede, o que não pode ter lugar para o isolamento e sentimento de alienação durante o período de trabalho.

Estas manifestações além de refletir na relação homem/trabalho também refletem na relação homem/lazer por gerar a perda de interesse pelo trabalho e pelo lazer.

A pesquisa constatou que $61,3 \%$ dos profissionais não têm momentos de lazer rotineiramente, alguns relatam a falta de tempo e horas extras que realizam, outros relatam falta de interesse para viagens, cinema ou outros tipos de diversão.

A evolução histórica a cerca do lazer e tempo livre fez com que a coexistência do tema gerasse uma definição dos termos considerada, por muitos autores, mais completa para o fenômeno. Dumazedier ${ }^{13}$ relaciona inicialmente o lazer com liberação e prazer, trazendo três funções diferentes: a do descanso, liberando a fadiga; do divertimento, recreação e entretenimento, ligada ao tédio e do desenvolvimento que se relaciona ao desenvolvimento da personalidade dependente da autonomia do pensamento e da ação cotidiana.

O lazer é um conjunto de ocupações às quais o indivíduo pode entregar-se de livre vontade, seja para repousar, seja para divertir-se, recrear-se e entreter-se ou ainda para desenvolver sua informação ou formação desinteressada, sua participação social voluntária ou sua livre capacidade criadora após livrarse ou desembaraçar-se das obrigações familiares e sociais ${ }^{13}$.

As atividades de lazer podem atingir os campos intelectuais, artísticos, físicos, manuais, turísticos e sociais, existindo diferentes buscas pelo prazer, associados ao imaginário, à realidade, à busca por informação, às práticas esportivas, à capacidade de manipulação, transformando objetos para lidar com a natureza e à quebra de rotina ou no relacionamento e convivência social ${ }^{14}$. Atualmente em uma sociedade capitalista percebemos que o lazer pode estar ligado ao consumo, por isso a cada dia observamos a busca desenfreada para satisfação dos desejos ligados a este fenômeno, porém muitas vezes estes desejos não são atingidos devido à exaustão profissional e cansaço excessivo.

Tempus, actas de saúde colet, Brasília, 11(4), 185-196, dez, 2017. Epub Ago/2018 ISSN 1982-8829 


\section{Outras Manifestações}

Para melhor entendimento estas manifestações foram divididas em aspectos positivos e negativos.

Foram encontrados nessa pesquisa, além das manifestações que se relacionam com a síndrome de Burnout na forma física, comportamental, psicológica e defensiva conforme a classificação de Teixeira ${ }^{15}$ e Pereira ${ }^{16}$, outros sinais e sintomas que também se relacionam com a síndrome e que estão em conformidade com a classificação feita pelo Ministério da Saúde ${ }^{17}$. Estas manifestações foram entendidas como negativas.

Outras manifestações consideradas positivas foram assim classificadas por não se relacionarem com o esgotamento emocional, mas por representar pensamentos positivos que combatem a insegurança e favorecem a auto-confiança ${ }^{18}$.

Dentre as manifestações negativas, destacaram-se o cansaço excessivo representado por 17,8\% dos profissionais, sentimento de esgotamento $(17,1 \%)$ e desmotivação $(11,1 \%)$. Estes resultados reforçam aspectos da síndrome de Burnout que afetam de forma negativa o cotidiano do serviço fazendo com a qualidade da assistência prestada ao cliente não seja satisfatória.

As manifestações vistas como positivas por não estarem relacionadas com a síndrome foram: o sentimento de esperança na ocorrência de mudanças que contribuam para qualificar a assistência e melhorar as condições de trabalho (18,3\%); de companheirismo (18,3\%), que está presente em situações de dificuldade e de limitações dos colegas; de compreensão $(18,3 \%)$ quanto à realidade da unidade e dificuldades existentes uma vez que grande parte delas depende de outros níveis de gerenciamento; atitude de superação (18,3\%) demonstrada com relatos de dificuldade no trabalho superadas por meio de adaptações pessoais; sentimento de domínio próprio $(17,1 \%)$ constatado frente a situações de iminente perca de controle emocional e, por fim, sentimento de ânimo e motivação $(9,7 \%)$ para com a possibilidade de melhora no atendimento. Algumas manifestações positivas estão apresentadas abaixo:

\footnotetext{
“Antigamente não tinha acolhimento, o povo morria na nossa frente, fazíamos quarenta curativos por dia, vivia com dores na coluna, (...) a saúde pública melhorou, se o PSF assumir, melhor, pode aliviar mais, porque se atendêssemos somente pacientes de emergência mesmo não seria tão estressante" (E9)
}

"Nunca perdi o controle não sempre me controlo" (TE7)

"Os colegas ajudam aí agente consegue trabalhar em equipe” (TE3)

Esta evidência é significativa por refletir diferentes visões dentro da enfermagem, não abordando apenas aspectos negativos, mas demonstrando sentimentos de enfrentamento a tudo aquilo que oferece risco ou ameaça ao controle emocional e à qualidade do serviço profissional. 
De acordo com a pesquisa apresentada foram encontrados 27 sinais e sintomas que fazem parte da síndrome de Burnout, sendo que estes sinais comuns apareceram aproximadamente 372 vezes durante a entrevista.

Os resultados evidenciaram que os entrevistados possuíam algum indício de Burnout, sendo que $45,1 \%$ estão na fase inicial, 35,5\% possuem uma possibilidade de desenvolver Burnout, em 9,7\% a Burnout começa a se instalar e 9,7\% se encontram na fase considerável de Burnout. Assim sendo, os sinais e sintomas da exaustão física e psicológica identificados nos profissionais da equipe de enfermagem do hospital estão relacionados com a síndrome.

\section{CONSIDERAÇÕES FINAIS}

O presente estudo discute aspectos importantes que diversas equipes de enfermagem da área da urgência e emergência e outras áreas vivenciam diariamente. É notório que pesquisas sobre fisiopatologia das doenças são de extrema importância, pois objetiva a busca pela saúde das pessoas, por melhores formas de tratamento das patologias e de promover à saúde, contudo também devemos nos preocupar com o cuidador que, se não for considerado de forma correta, poderá se tornar um doente.

Atualmente percebemos grande número de profissionais doentes cuidando de doentes, o que prejudica a assistência prestada. Os gestores das unidades devem se preocupar com a saúde do trabalhador, com a humanização das relações promovendo assim, mais credibilidade dos hospitais e da saúde pública.

Esta pesquisa atingiu, satisfatoriamente, aos objetivos propostos, podendo contribuir para pesquisas na área da urgência/emergência e enfermagem, saúde do trabalhador, gestão de unidade hospitalar além de enriquecer o ensino e a pesquisa.

\section{REFERÊNCIAS BIBLIOGRÁFICAS}

1- AMADOR, J.C. et al. Síndrome de burnout em profissionais de enfermagem. Revista OMNIA Saúde, Adamantina (SP), v. 10, 2013.

2- JODAS, D. A; HADDAD, M. C. L. 2008. Síndrome de Burnout em trabalhadores de enfermagem de um pronto socorro de hospital universitário. Acta Paul Enferm. 2009; 22 (2):192-7

3- DIAS S.; QUEIRÓS, C.; CARLOTTO, M. S. Síndrome de burnout e fatores associados em profissionais da área da saúde: um estudo comparativo entre Brasil e Portugal. Aletheia n.32 Canoas, 2010.

4- MOREIRA, D. S. et al. Prevalência da síndrome de burnout em trabalhadores de 
enfermagem de um hospital de grande porte da Região Sul do Brasil. Cad. Saúde Pública, Rio de Janeiro, 25(7):1559-1568, jul, 2009.

5- JODAS, D. A.; HADDAD, M. C. L. SÌndrome de Burnout em trabalhadores de enfermagem de um pronto socorro de hospital universitário. Disponível em: < http://www.scielo. br/pdf/ape/v22n2/a12v22n2.pdf> Acessado em: 15/09/2015.

6- SANTOS FILHO , J. C. Pesquisa quantitativa versus pesquisa qualitativa: o desafio paradigmático. Em: SÁNCHEZ GAMBOA, S. (org.). Pesquisa educacional: quantidadequalidade. 4.ed., São Paulo: Cortez, 2001.

7- MASLACH C, JACKSON S. E. Maslach Burnout inventory. 2. ed. Palo Alto: Consulting Psychologists Press; 1986.

8- BRASIL. Ministério da Saúde. Secretaria de Atenção à Saúde. Departamento de Atenção Básica. Envelhecimento e saúde da pessoa idosa / Ministério da Saúde, Secretaria de Atenção à Saúde, Departamento de Atenção Básica - Brasília : Ministério da Saúde, 2006.

9- FIRME, E. ALVES, J.; ROQUE,R. A. Síndrome de Burnout. 2007. Disponível em https:// moodle.fct.unl.pt/pluginfile.php/54757/mod_resource/content/0/extracurricular_activities/ sindrome burnout trab final.pdf acessado em 09 de Janeiro de 2017.

10- DRUMMONDI, B. L. C., RADICCHIII, A. L. A., GONTIJOII, E. C. D. Fatores sociais associados a transtornos mentais com situações de risco na atenção primária de saúde. Rev bras epidemiol suppl D.S.S. 68-80, 2014.

11- FRANÇA, F. M., FERRARI, R. Síndrome de Burnout e os aspectos sócio-demográficos em profissionais de enfermagem Acta paul. Enferm. São Paulo, 2012. 25 (5).

12- BRASIL. Ministério da Saúde. Secretaria de Atenção à Saúde. Política Nacional de Humanização da Atenção e Gestão do SUS. Acolhimento e classificação de risco nos serviços de urgência. Brasília, 2009. 56 p.

13- DUMAZEDIER, J. Lazer e cultura popular. São Paulo: Perspectiva, 1973.

14- BARBOSA, T. P.; SILVA, O. V. Origens e significados do lazer. Revista científica eletrônica de turismo - ISSN: 1806-9169 Ano VIII - Número 14. 2011.

15- TEIXEIRA, M. O burnout e os enfermeiros. Rev. Saúde mental e psiquiátrica. 2008. Disponível em: http://br.monografias.com/trabalhos3/burnoutenfermeiros/burnout-enfermeiros2. shtml\#preven, Acesso em 10 Maio. 2016.

16- PEREIRA, A. M. T.B. Burnout: quando o trabalho ameaça o bem-estar do trabalhador. ISSN 1982-8829 Tempus, actas de saúde colet, Brasília, 11(4), 185-196, dez, 2017. Epub Ago/2018 
$196 / /$

1. ed. São Paulo : casa do psicólogo, 2002

17- BRASIL, Ministério da Saúde. Doenças relacionadas ao trabalho: manual de procedimentos para os serviços de saúde. Brasília, 2001.

18- SOUSA, A. D.,O outro lado da enfermagem. Ordem dos enfermeiros.In. jornal madeira, 2014. Disponível em :http://www.ordemenfermeiros.pt/sites/madeira/informacao/Documents/ ARTIGOS\%202014/o\%20outro\%201ado\%20\%20\%20\%20mar\%C3\%A7o\%202014.pdf acessado em 09 de Janeiro de 2017.

Artigo apresentado em 17/03/2017

Artigo aprovado em 15/09/2017

Artigo publicado no sistema em 30/03/2018 